\title{
CONTRIBUIÇÕES DO PLANO DE PARTO E ESTRATÉGIAS PARA INSERÇÃO NO PRÉ-NATAL: REVISÃO NARRATIVA ${ }^{1}$ CONTRIBUTIONS TO THE BIRTH PLAN AND STRATEGIES FOR PRENATAL INSERTION: NARRATIVE REVIEW
}

\section{Carla Cristiana Costodio Pereira ${ }^{2}$, Liliane Jaci Roos Buttow ${ }^{3}$, Luiza Cremonese ${ }^{4}$, Geórgia Figueira Rampelotto ${ }^{5}$, Laís Antunes Wilhelm ${ }^{6}$ e Camila Nunes Barreto ${ }^{7}$}

\begin{abstract}
RESUMO
O estudo objetivou descrever as estratégias para a construção de um plano de parto na consulta de pré-natal realizada por enfermeiros. Trata-se de um estudo bibliográfico narrativo. O levantamento de dados ocorreu no mês de outubro de 2019, compreendendo produções científicas, entre os anos de 2011 e 2019, os dados foram coletados nas bases de dados da Literatura Latino Americana e do Caribe em Ciências da Saúde e na Medical Literature Analysis and Retrieval System Online; manuais do Ministério da Saúde e da Organização Mundial da Saúde. O plano de parto é importante para a condução do trabalho de parto e parto em si, pois a gestante adquire confiança, protagoniza a vivência junto à sua família e tem seus direitos e decisões respeitados pelos profissionais de saúde. É necessário o desenvolvimento de pesquisas sobre plano de parto, visto a baixa quantidade existente na literatura, a fim de promover sua inserção como rotina na consulta de pré-natal.
\end{abstract}

Palavras-chave: Cuidado pré-natal; Enfermagem; Parto; Atenção Primária à Saúde; Educação em Saúde.

\section{ABSTRACT}

The study aimed to describe the strategies to design a birth plan during the prenatal appointament performed by nurses. This is a narrative bibliographic study. The data survey took place in October 2019, by analysing scientific productions, between the years 2011 and 2019, the data were collected from databases of Latin American and Caribbean Literature in Health Sciences and in Medical Literature Analysis and Retrieval System Online; manuals of the Ministry of Health and the World Health Organization. The birth plan play an important role for the conduct of labor and the delivery itself. It helps the pregnant woman to gain confidence, to explain to her family and to the stafss how would she like to have her rights and decissions respected during the procedure. The objective of this study to support the Birth Plan the inclusion in the Prenatal consultation. Therefore, futher studies on birth Plan are needed due the lack of information avalilable about this subject.

\section{Keywords: Prenatal care; Nursing; Parturition; Primary Health Care; Health Education.}

${ }^{1}$ Estudo oriundo de Trabalho de Conclusão de Curso de Enfermagem da Universidade Luterana do Brasil - Campus Cachoeira do Sul.

${ }^{2}$ Enfermeira. Graduada pela Universidade Luterana do Brasil - Campus Cachoeira do Sul. Atua no Hospital Montenegro 100\% SUS. E-mail: carlacristiana32@gmail.com

${ }^{3}$ Enfermeira na Secretaria Municipal de Saúde do município de Cachoeira do Sul. E-mail: lilianeroos@yahoo.com.br

${ }^{4}$ Enfermeira. Graduada pela Universidade Federal de Santa Maria. Doutora em Enfermagem pelo PPGENF/UFSM. Docente do Curso de Enfermagem - Ulbra Cachoeira do Sul. E-mail: lu_cremonese@hotmail.com

${ }^{5}$ Enfermeira na Secretaria Municipal de Saúde do município de Cachoeira do Sul. E-mail: ge.rampelotto@gmail.com

${ }^{6}$ Enfermeira. Graduada pela Universidade Federal de Santa Maria. Doutora em Enfermagem pelo PPGENF/UFSM. Docente do Departamento de Enfermagem da Universidade Federal de Santa Catarina E-mail: laiswilhem@gmail.com ${ }^{7}$ Enfermeira. Graduada pela Universidade Federal de Santa Maria. Doutoranda em Enfermagem pelo PPGENF/UFSM. Docente do Curso de Enfermagem - Ulbra Cachoeira do Sul. E-mail: camilabarreto_6@msn.com 


\section{INTRODUÇÃO}

O cuidado pré-natal se configura como o primeiro passo para qualificação do parto e nascimento. Ele possibilita a prevenção de complicações causadoras de morbimortalidade materna e perinatal. Além disso, aborda diferentes questões relacionadas à evolução da gravidez e o preparo da mulher e da família para cada etapa do ciclo gravídico-puerperal (MOUTA et al., 2017).

O acompanhamento do pré-natal representa um elemento essencial na assistência às gestantes e suas famílias, pois assegura um melhor epílogo materno e neonatal. O cuidado prestado durante as consultas visa auxiliar na evolução da gestação, diagnosticar e tratar morbidades, tornando-se uma oportunidade para que o enfermeiro fortaleça também, ações de educação em saúde (GONÇALVES et al., 2017).

Portanto, a preparação da gestante para o momento do parto, bem como o acompanhamento do desenvolvimento do ciclo gravídico, firmam-se como imprescindíveis, uma vez que minimizam o aparecimento de problemas clínicos e operam preventivamente, quando necessário. Ainda, contribuem para esclarecimentos de dúvidas acerca da gestação e a experiência do parto, a qual necessita ser contemplada durante o pré-natal (GUEDES et al., 2017).

Nas últimas décadas, conquistaram-se mudanças relacionadas ao parto e ao nascimento. No contexto das políticas públicas e programas de saúde direcionados ao parto e nascimento, destaca-se o Programa de Humanização do Pré-Natal e Nascimento (PHPN), que foi criado no ano de 2000 com o propósito de orientar as instituições de saúde a adotar medidas para a prevenção de morbimortalidade e promoção da saúde para a mulher e criança (BRASIL, 2002a). Vale destacar ainda que, em 2005, foi sancionada a lei $n^{\circ} 11.108 / 95$, a lei do acompanhante, que permite que a gestante escolha uma pessoa, seja homem ou mulher, parente ou não, para lhe acompanhar em todo o processo de trabalho de parto e parto. No ano de 2011, a partir da Portaria n ${ }^{\circ}$ 1459, foi lançado pelo Ministério da Saúde (MS) o Programa Rede Cegonha, buscando estabelecer mudanças na assistência do pré-natal, trabalho de parto, parto e nascimento, com enfoque em organizar, estruturar e qualificar a atenção à gestante e recém-nascido (BRASIL, 2011b).

Nessa direção, destaca-se o papel do enfermeiro, a fim de assegurar à gestante uma assistência integral e de qualidade, munindo-a de informações e orientações capazes de torná-la protagonista durante todo o processo. Desta forma, para que haja a garantia de êxito na atenção do pré-natal, é preciso que, além de uma satisfatória avaliação de riscos, ocorra a qualificação do profissional com foco no cuidado humanizado. $\mathrm{O}$ próprio MS coloca a humanização na atenção à gestante como garantia de um padrão de acesso e qualidade aos serviços de saúde (BARROS et al., 2017).

Nesse ínterim, destaca-se o Plano de Parto (PP), visto que ele se mostra como garantia e incentivo ao processo de humanização do nascimento, além de ser parte do pressuposto do parto como um evento fisiológico e afetivo, em que as escolhas da mulher e de sua família necessitam ser respeitadas. 
Trata-se de um documento legal escrito pelas gestantes após o recebimento de informações sobre a gestação e o processo de parto, respeitando seus valores, desejos pessoais e expectativas criadas sobre o parto durante a gravidez. É utilizado para orientar a atenção de saúde prestada à gestante neste processo (BARROS et al., 2017)

O primeiro PP foi preparado por Sheila Kitzinger, nos Estados Unidos, no ano de 1980, enunciando que parto é um processo fisiológico pelo qual a grande maioria das mulheres irá passar ao longo de suas vidas, não podendo tornar-se algo patológico e medicalizado. Assim, desde 1996, o PP é recomendado pela Organização Mundial de Saúde (OMS) como mecanismo de incentivo às mulheres na busca de informações qualificadas. (BRASIL, 2014c).

A importância do PP está condicionada ao princípio bioético da autonomia e empoderamento, uma vez que se encontra em uma posição evidenciada dentre as condutas que devem ser estimuladas durante a gestação pela OMS. O PP deve ser construído pela gestante em conjunto com os enfermeiros da Atenção Primária de Saúde, e discutido com os profissionais que irão realizar o atendimento hospitalar (MEDEIROS et al., 2019).

A pesquisa Nascer no Brasil - Inquérito Nacional sobre Parto e Nascimento apresenta indicações de que o modelo medicalizado predomina, com excesso de intervenções durante o parto (medicamentos para aceleração/indução do parto, anestesia, episiotomia), aliado à ocorrência de cesariana desnecessária. Essas situações figuram como parte das consequências do aumento na taxa de nascimento de bebês pré-termo. No Brasil, o índice de cesarianas chega a 52\%, sendo que no setor privado o percentual de cesarianas é de $88 \%$ dos nascimentos. No setor público as cesarianas chegam a 46\%, envolvendo serviços do Sistema Único de Saúde (SUS) e contratados do setor privado (LEAL et al., 2014). Esses dados alarmam para o fato de que o nascimento não é um acontecimento natural, pois são utilizadas intervenções que, muitas vezes, não vão ao encontro ao desejo da gestante, sendo que na maioria das situações ela nem é consultada sobre suas vontades ou informada a respeito das condutas que a equipe médica realiza durante o parto (LEAL et al., 2014).

A partir dessas considerações, ressalta-se a essencialidade de consolidar práticas efetivas para construção do plano de parto na atenção primária à saúde pelo enfermeiro e demais profissionais atuantes nesse contexto. Nessa direção, a questão de revisão do estudo foi: quais as estratégias para construção de um plano de parto na consulta de pré-natal realizada por enfermeiros? E objetivou descrever as estratégias para a construção de um plano de parto na consulta de pré-natal realizada por enfermeiros.

\section{MATERIAL E MÉTODOS}

Trata-se de um estudo bibliográfico, do tipo narrativo, sobre as produções científicas disponíveis acerca das estratégias para construção do plano de parto na consulta de pré-natal realizada por enfermeiros. Optou-se pelo estudo narrativo, pois, ao realizar uma busca prévia, verificou-se o 
número baixo de produções relacionadas à temática e a necessidade de explorá-la amplamente para fornecer subsídios para a prática de enfermagem.

Foram percorridas as seguintes etapas para execução do estudo: estabelecimento da questão de revisão, definição dos critérios de seleção, coleta de dados, categorização dos estudos, interpretação dos resultados e síntese do conhecimento, culminando com a correlação da literatura científica (BRUM et al., 2016). Nessa direção, a questão revisão do estudo foi: quais as estratégias para construção de um plano de parto na consulta de pré-natal realizada por enfermeiros?

O levantamento de dados ocorreu no período de outubro de 2019. Como critérios de seleção, foram estabelecidas produções que responderam à questão norteadora e fornecessem subsídios para construção do plano de parto na prática do profissional enfermeiro.

As buscas nas fontes de dados foram realizadas na Literatura Latino Americana e do Caribe em Ciências da Saúde (LILACS) e na Medical Literature Analysis and Retrieval System Online (MEDLINE); manuais do Ministério da Saúde e Organização Mundial da Saúde, estudos entre 2011 e 2019, nos idiomas inglês ou espanhol ou português. O marco temporal foi estabelecido em razão da criação da Rede Cegonha pelo Ministério da Saúde, no ano de 2011. A Rede Cegonha foi uma política de grande marco para qualificação das práticas associadas ao período de gravidez, parto e nascimento (BRASIL, 2011b). Para a realização da busca dos artigos e materiais científicos, foram utilizadas as seguintes palavras-chave e estratégias de busca: "plano de parto" and "enfermagem"; "plano de parto" or "parto humanizado" e ("birth plan" and "nursing" or "humanized birth").

O corpus final do estudo foi de quatorze publicações, sendo 11 artigos e dois manuais/diretrizes do Ministério da Saúde e um documento da Organização Mundial da Saúde. A organização da coleta de dados foi realizada na forma de quadro sinóptico, o que possibilitou assentar o material encontrado e permitir um estudo subsequente. O quadro 1 a seguir apresenta os quatorze estudos selecionados para essa revisão.

Quadro 1 - Produções obtidas nas fontes de dados acerca das estratégias para a construção de um plano de parto na consulta de pré-natal realizada por enfermeiros.

\begin{tabular}{|c|c|l|l|}
\hline Material & Referência & \multicolumn{1}{|c|}{ Objetivo } & \multicolumn{1}{|c|}{ Conclusão } \\
\hline M1 & 1 & $\begin{array}{l}\text { Identificar o conhecimento de enfermeiras de } \\
\text { um município da fronteira oeste do RS, sobre } \\
\text { plano de parto. }\end{array}$ & $\begin{array}{l}\text { O estudo possibilitou aprofundamento na } \\
\text { temática, propiciando algumas reflexões junto a } \\
\text { profissionais que estavam inseridos na Atenção } \\
\text { Primária à Saúde }\end{array}$ \\
\hline M2 & 2 & Documento ministerial & Documento ministerial \\
\hline M3 & 3 & Documento ministerial & Documento ministerial \\
\hline M4 & 7 & $\begin{array}{l}\text { Formalizar um modelo de referência para o plano } \\
\text { de parto informatizado e identificar um conjunto } \\
\text { de arquétipos para representar seus conceitos. }\end{array}$ & $\begin{array}{l}\text { Espera-se que esta proposta seja um ponto de } \\
\text { partida, após sua validação na prática clínica, } \\
\text { para viabilizar o acesso ao plano de parto em } \\
\text { canais informatizados do pré-natal ao parto. }\end{array}$ \\
\hline
\end{tabular}




\begin{tabular}{|c|c|c|c|}
\hline M5 & 8 & $\begin{array}{l}\text { Conhecer, analisar e descrever a situação atual dos } \\
\text { planos de parto e nascimento no contexto estudado, } \\
\text { comparando o processo de parto e sua finalização } \\
\text { entre as mulheres que apresentaram e as que não } \\
\text { apresentaram um plano de parto e nascimento. }\end{array}$ & $\begin{array}{l}\text { Os planos de parto e nascimento influenciam } \\
\text { positivamente o processo de parto e sua finali- } \\
\text { zação. São necessárias políticas sanitárias para } \\
\text { aumentar o número de planos de parto e nasci- } \\
\text { mento apresentados nos hospitais estudados. }\end{array}$ \\
\hline M6 & 9 & $\begin{array}{l}\text { Caracterizar os desejos e expectativas de ges- } \\
\text { tantes descritos em um plano de parto. }\end{array}$ & $\begin{array}{l}\text { Cabe aos profissionais de saúde proporcionar } \\
\text { informações que contribuam para a tomada de } \\
\text { decisão da mulher. Acredita-se que, ao adquirir } \\
\text { conhecimento e receber estímulo da equipe de } \\
\text { saúde, a gestante realizará escolhas informadas } \\
\text { e se aproximará de um atendimento qualificado } \\
\text { e humanizado. }\end{array}$ \\
\hline M7 & 13 & $\begin{array}{l}\text { Analisar as repercussões da utilização do Plano } \\
\text { de Parto no processo de parturição a partir da } \\
\text { produção científica nacional e internacional. }\end{array}$ & $\begin{array}{l}\text { As publicações analisadas justificam a im- } \\
\text { plementação clínica do Plano de Parto por se } \\
\text { configurar como tecnologia potencializadora } \\
\text { de cuidados humanizados e satisfação materna. } \\
\text { Persistem desafios relacionados à adesão das } \\
\text { mulheres ao instrumento e apoio profissional } \\
\text { para melhorar o cumprimento destes. }\end{array}$ \\
\hline M8 & 14 & $\begin{array}{l}\text { Analisar como o plano de parto propiciou o } \\
\text { empoderamento feminino durante o trabalho de } \\
\text { parto e parto. }\end{array}$ & $\begin{array}{l}\text { Destacou-se a importância do enfermeiro } \\
\text { obstétrico atuando em partos e utilizando o } \\
\text { plano de parto como uma tecnologia não inva- } \\
\text { siva. A construção do plano de parto durante } \\
\text { o pré-natal contribui para o desenvolvimento } \\
\text { favorável do trabalho de parto. }\end{array}$ \\
\hline M9 & 15 & $\begin{array}{l}\text { Verificar o conhecimento de estudantes sobre o } \\
\text { plano individual de parto e conhecer sua opinião } \\
\text { a respeito da utilização dessa estratégia de ensino- } \\
\text {-aprendizagem e das boas práticas obstétricas. }\end{array}$ & $\begin{array}{l}\text { Além de conhecerem o plano de parto e aplicá-lo, } \\
\text { os estudantes o consideram muito relevante para } \\
\text { o ensino e a aprendizagem das boas práticas } \\
\text { obstétricas. }\end{array}$ \\
\hline M10 & 16 & $\begin{array}{l}\text { Conhecer a compreensão dos profissionais de } \\
\text { saúde de uma unidade hospitalar obstétrica } \\
\text { referente às boas práticas de atenção ao parto e } \\
\text { ao nascimento preconizadas pela Organização } \\
\text { Mundial da Saúde. }\end{array}$ & $\begin{array}{l}\text { As boas práticas, além de possibilitarem o } \\
\text { repensar do modelo obstétrico e contribuírem } \\
\text { para organizar a rede de atenção à saúde mater- } \\
\text { no infantil a fim de garantir acesso, acolhimento } \\
\text { e resolutividade, estimulam o protagonismo da } \\
\text { mulher em suas múltiplas dimensões. }\end{array}$ \\
\hline M11 & 17 & $\begin{array}{l}\text { Analisar a percepção das mulheres que reali- } \\
\text { zaram o plano de parto sobre a experiência de } \\
\text { parto, os significados do plano de parto, seus } \\
\text { elementos constituintes e a relação do plano de } \\
\text { parto com o trabalho de parto e parto. }\end{array}$ & $\begin{array}{l}\text { Observou-se relação direta com a realização do } \\
\text { plano de parto e a experiência do parto positiva. } \\
\text { Destaca-se a importância da utilização do plano } \\
\text { de parto como uma tecnologia que favorece } \\
\text { a experiência positiva do parto. A construção } \\
\text { do plano pelas mulheres durante o pré-natal e } \\
\text { a realização dele por parte da equipe de saúde } \\
\text { contribuíram para o desenvolvimento favorável } \\
\text { do trabalho de parto. }\end{array}$ \\
\hline M12 & 18 & $\begin{array}{l}\text { Refletir sobre a importância do plano de parto } \\
\text { na assistência de enfermagem, visando à auto- } \\
\text { nomia da mulher. }\end{array}$ & $\begin{array}{l}\text { Evidencia-se, nesse estudo, a importância do } \\
\text { uso do plano de parto pela enfermagem como } \\
\text { instrumento de empoderamento da mulher. Ele é } \\
\text { uma ferramenta que deve ser utilizada, visando à } \\
\text { garantia de uma assistência de enfermagem huma- } \\
\text { nizada, de qualidade e isenta de danos à mulher. }\end{array}$ \\
\hline
\end{tabular}




\begin{tabular}{|c|c|l|l|}
\hline \multirow{2}{*}{} & & $\begin{array}{l}\text { Descrever características sociodemográficas e } \\
\text { identificar as informações precedentes sobre } \\
\text { o plano de parto das gestantes atendidas em } \\
\text { unidades básicas de saúde (UBS), no município } \\
\text { de Belo Horizonte/MG. }\end{array}$ & $\begin{array}{l}\text { Profissionais de saúde envolvidos na assistên- } \\
\text { cia à gestante precisam proporcionar espaços } \\
\text { para a discussão do plano de parto, facilitando } \\
\text { a comunicação entre profissionais e gestantes, } \\
\text { contribuindo para o fortalecimento da autono- } \\
\text { mia e tomada de decisão no processo de parto e } \\
\text { nascimento. Acredita-se que o conhecimento do } \\
\text { contexto de vida dessas gestantes e da prática } \\
\text { realizada pelos profissionais auxilia no direcio- } \\
\text { namento de uma assistência específica e permitir } \\
\text { traçar ações que atendam ao perfil dessa clientela. }\end{array}$ \\
\hline M14 & 20 & Documento ministerial & Documento ministerial \\
\hline
\end{tabular}

Fonte: Elaborado pelos autores.

\section{RESULTADOS}

Ao caracterizar as quatorze produções, ressalta-se que os materiais encontrados são provenientes da área da saúde e, na sua maioria, foram escritos por enfermeiros. O maior contingente dos estudos selecionados foi publicado no estado do Rio Grande do Sul (5), seguido de Brasília (3), e estados da Bahia, Rio de Janeiro, Minas Gerais e São Paulo (1 publicação por estado). Ainda se acrescenta dois documentos ministeriais e um documento da OMS.

O cenário dos estudos, provenientes de artigos originais, foram em unidades de Atenção Básica, envolvendo grupos de gestantes e profissionais da saúde, como enfermeiros, técnicos de enfermagem e médicos. Grande parte das publicações escolhidas são do ano de 2017 (5), seguidos pelos anos de 2019 (3), 2018 (2), tendo ainda nos anos de 2011, 2013, 2014 e 2015 com uma publicação correspondente a cada um. Quanto ao desenho dos estudos selecionados, cinco estudos eram qualitativos, quatro estudos eram quantitativos, um era estudo de caso, um era revisão integrativa da literatura, dois manuais ministeriais e um documento da OMS.

A partir da análise das produções, foram constituídas duas categorias temáticas: "Contribuições do plano de parto para mulher e sua família" e "Elementos constitutivos do plano de parto no pré-natal de risco habitual”. Além disso, a fim de contribuir com uma melhor explanação dos resultados encontrados, foram incluídas figuras representativas para exemplificar os achados.

\section{CONTRIBUIÇÕES DO PLANO DE PARTO PARA MULHER E SUA FAMÍLIA}

O PP é um instrumento que contribui para a qualificação da assistência de enfermagem, pautada nos direitos das mulheres e de sua família, o que repercute na humanização do cuidado. Ainda, trata-se de uma técnica que deve ser amplamente estimulada durante a gestação e que obedece às normas internacionais da OMS. Ademais, destacam-se os ganhos no uso do PP, como o empoderamento da mulher e minimização de danos à sua saúde (M6, M8, M12, M14). 
O PP é considerado uma tecnologia não invasiva de cuidado, uma vez que busca reaver o papel da mulher como protagonista no momento do trabalho de parto e parto, neutralizando as técnicas do modelo hospitalocêntrico (M8, M11, M12). A mulher é protagonista na construção do PP, fazendo com que sejam respeitadas suas decisões e buscando um maior suporte para trabalhar com as mudanças que vem ocorrendo na sua vida. Elaborar um PP oferta o empoderamento às gestantes no processo de parturição, considerando que todas se sentem protagonistas dos seus partos (M4, M6, M7, M9, M11, M13).

O enfermeiro figura como uma peça importante nessa construção, seu papel enquanto profissional da saúde inicia no pré-natal, momento em que o PP é apresentado à gestante e sua construção instigada (M8, M9, M13). Nesse momento, cabe a ele prestar apoio à gestante, bem como assegurar respeito, segurança, privacidade e permitir que ela se fortaleça no trabalho de parto (M6, M8, M10, M11, M12). Essa construção, quando feita em conjunto com o profissional de saúde, possibilita a estruturação de um vínculo entre enfermeiro-gestante, além de garantir que sejam respeitadas as práticas alicerçadas em evidências científicas.

A construção de um PP soa como imprescindível para a condução do trabalho de parto em si, pois a gestante adquire confiança sobre o processo de nascimento quando está ciente e tem conhecimento do que pode acontecer durante o trabalho de parto e parto (M1, M5, M8, M11, M12). Observa-se também que conforme o cumprimento do PP aumenta, as taxas de cesáreas consequentemente diminuem e melhoram os resultados nos testes de Apgar no primeiro minuto e no $\mathrm{pH}$ do cordão umbilical (M3, M7). A oferta de ações educativas promovendo a troca de vivências e experiências sobre o parto de maneira positiva contribuiu para um melhor entendimento e construção do PP pelas gestantes. No entanto, mesmo que a inclusão do PP nos protocolos assistenciais seja algo positivo, a elaboração deste não pode ser algo imposto pelo enfermeiro. O PP precisa ser visto como um ensejo em que o profissional da saúde conceitue o seu trabalho e a mulher garanta o respeito de seus direitos e desejos, culminando na condição de excelência na assistência para a mãe e o filho, consolidando as relações familiares (M3, M4, M5, M8, M9, M10, M12, M13).

No que se refere aos benefícios do PP, podemos citar o aumento da comunicação entre gestantes e profissionais de saúde, estimulando discussões, a consciência das opções disponíveis e o senso de controle durante o episódio da parturição (M3, M6, M7, M12, M13). Destaca-se ainda que o PP também pode ser elaborado na carteira da gestante, uma vez que se encontra disponível na versão impressa do cartão de pré-natal. Aliado a isso, o PP informatizado, em modelo de arquétipo, surgiu como uma forma de viabilizar seu acesso em canais informatizados do pré-natal ao parto, podendo ser acessado em todo território nacional e internacional (M4).

Tal ideia se mostrou como algo inovador, uma vez que os profissionais de saúde consideram o uso de tecnologias da informação em saúde como uma estratégia eficaz na melhoria da qualidade da assistência ofertada à gestante. Essa estratégia tende a aprimorar a continuidade dos registros, 
incentivar a completude dos dados, fornecer apoio à decisão e reduzir a incidência de erros clínicos na área obstétrica (M3, M4, M7, M9).

Figura 1 - Representação acerca da categoria 1 - Contribuições do plano de parto para mulher e sua família.

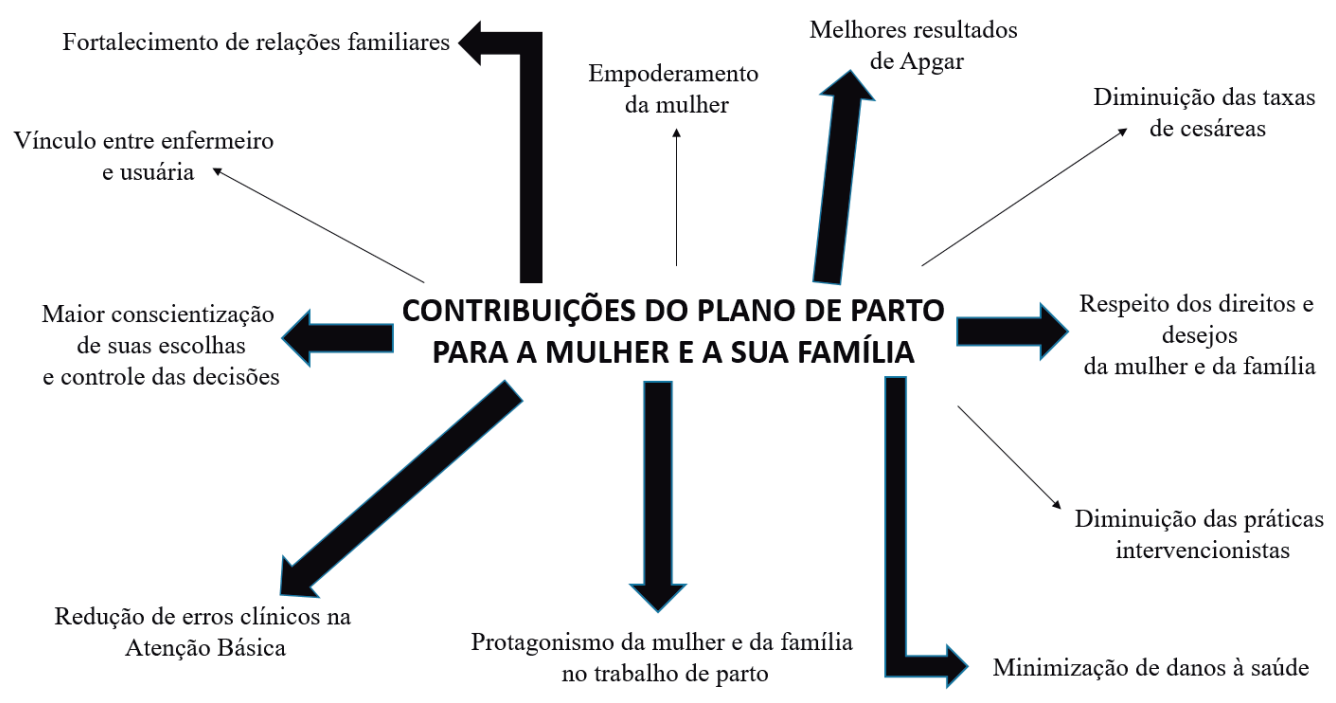

Fonte: Elaborado pelos autores.

\section{ELEMENTOS CONSTITUTIVOS DO PLANO DE PARTO NO PRÉ-NATAL DE RISCO HABITUAL}

O ato de preparação da mulher e sua família é o mesmo que oportuniza a ela o seu empoderamento, para que possa empenhar-se na busca pelo seu direito à escolha informada. Estando preparadas, são capazes de vivenciar da melhor forma seus resultados físicos e emocionais quando envolvidas nas decisões sobre o seu parto, sendo capazes de agir de forma ativa e com autonomia, beneficiando a ocorrência de resultados positivos no processo (M6, M7, M9, M13).

O PP é um documento legal elaborado pelas gestantes tão logo recebido dos profissionais de saúde as informações sobre o processo da gestação e parturição. Por se tratar de um fator favorável para o conhecimento das parturientes sobre seus direitos e boas práticas de atenção, ele é o eixo de relação entre a gestante e o profissional de saúde. Ele contém os valores e desejos pessoais de cada mulher e é utilizado para orientar a atenção de saúde prestada ao longo de todo o processo (M3, M4, M7, M10, M11, M12).

De acordo com a visão das gestantes, o PP engloba componentes que dizem respeito aos seus direitos garantidos. Esses, mesmo sendo recomendados pela OMS e apoiadas pelo MS, muitas vezes permanecem ignorados (M1, M3, M11, M12, M14).

O PP inclui a presença de acompanhante, bem como a presença de pessoas da rede de apoio da mulher que estarão presentes no momento do processo de parturição. Ressalta-se a importância de conhecer a maternidade de referência para o seu parto, a inclusão da ingesta alimentar e informações 
sobre os procedimentos a serem desenvolvidos durante o trabalho de parto, não haver intervenções desnecessárias, uso de métodos de alívio de dor tanto farmacológicos como não farmacológicos, cuidados desejados com o recém-nascido, intervenções médicas diante de possíveis intercorrências, observações culturais, música ambiente, banho/presença de chuveiro ou piscina, posição para o trabalho de parto e parto, corte do cordão umbilical após cessar a pulsação, presença e amamentação de recém-nascido no pós-parto (M1, M3, M7, M11, M12, M14).

$\mathrm{O}$ PP apresentado pela mulher necessita ser lido e discutido, além de ser adaptado à realidade local da maternidade de referência. Diante disso, reconhecer a realidade local de assistência ao parto e nascimento é essencial a fim de oferecer orientações e condutas de acordo com a organização do local de assistência. Dessa maneira, leva-se em consideração limitações estruturais e de recursos humanos, os métodos e técnicas realizadas pelas instituições e quais os profissionais de saúde estão capacitados para ofertar o cuidado (M2).

Recomenda-se evitar interferências desnecessárias, assim como, a avaliação correta baseada em evidências científicas de fatores que influenciam na evolução do trabalho de parto e parto. Outro ponto importante, mesmo se a intervenção for necessária, no caso de alguma complicação, é que as mulheres sejam ouvidas e estejam informadas das condutas a serem adotadas. Destaca-se que as preferências e necessidades da mulher são os alicerces dessa construção (M14).

Diante disso, o PP construído pela mulher durante a sua gestação surge como uma forma de ponderar limites e possibilidades das opções específicas no momento do parto e pós-parto. Oportuniza ainda a reflexão e ponderação sobre o que a gestante está disposta ou não a assentir que seja executado no seu corpo, enaltecendo a confiança da parturiente na sua capacidade de parir e o direito de decidir sobre as suas escolhas (M4, M9, M13).

Figura 2 - Representação acerca da categoria 2 - Elementos constitutivos do plano de parto no pré-natal de risco habitual.

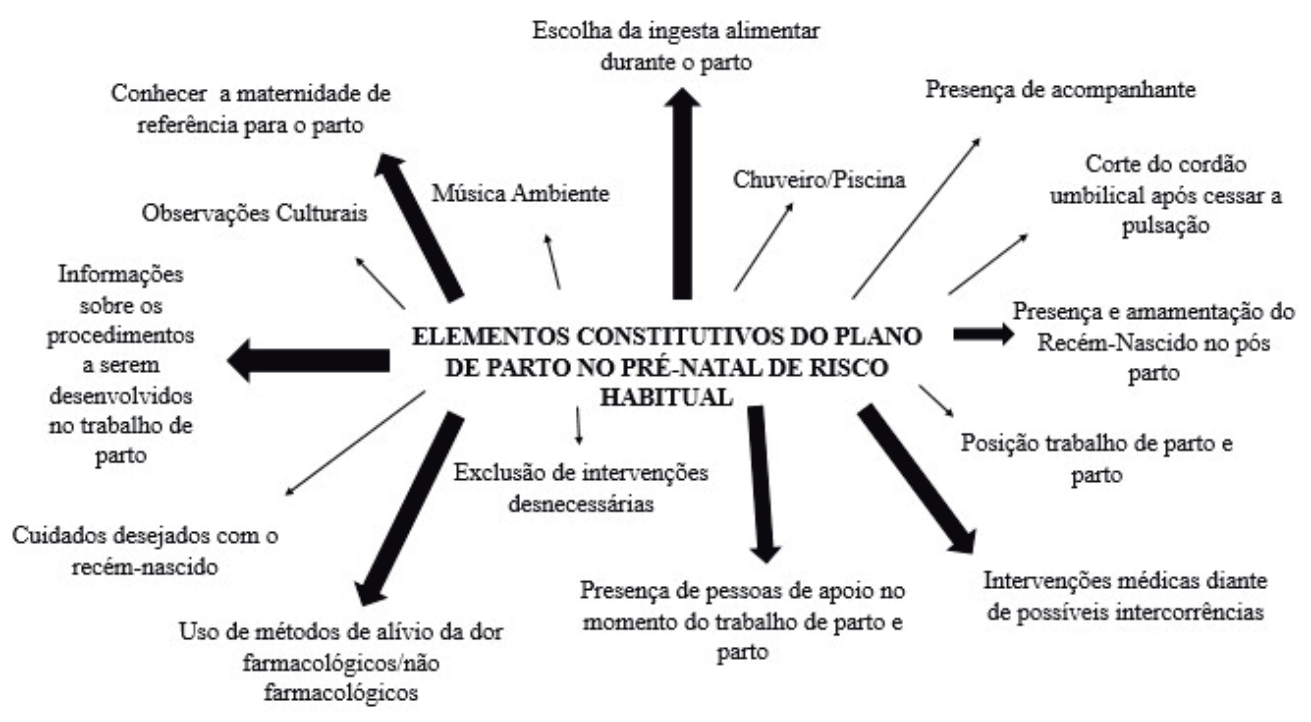

Fonte: Elaborado pelos autores. 


\section{DISCUSSÃO}

O PP é baseado na seguinte tríade: a restituição do protagonismo feminino, a visão do parto como um evento humano biopsicosociocultural e vinculação com a medicina baseada em evidências, trazendo o PP como uma tecnologia intensificadora dos cuidados humanizados à mulher e ao recém-nascido (MEDEIROS et al., 2019). Os resultados benéficos da sua aplicação estão ajustados às condutas recomendadas pela OMS, com o intuito de promover as boas práticas de atenção ao parto e nascimento em consonância com evidências científicas atuais, proporcionando melhor qualidade da atenção à saúde materna infantil (BARROS et al., 2017)

$\mathrm{O} P \mathrm{P}$ é um elemento educativo de grande efeito. Ele possibilita às gestantes o acesso a informações no que diz respeito a autodeterminação, direito de liberdade, privacidade, escolha individual e livre vontade, subvencionando a composição de conhecimentos indispensáveis para a determinação quanto ao parto e o nascimento da criança (SILVA et al., 2015).

A construção do PP não é um processo simples, sendo que na maioria das vezes as gestantes mostram dificuldades para entender as possibilidades de escolha que compõem o instrumento (NARCHI et al., 2019). Elaborar o PP durante a gestação e apresentá-lo no momento certo assegura, além de um parto com intervenções reduzidas, repercussões clínicas favoráveis como questões de ordem psicoemocionais. Nessa esteira de pensamento, o/um estudo refere que quando as mulheres se mostram mais preparadas, elas relatam confiança, autonomia, maior participação no processo parturitivo, ocasionando um resultado positivo da experiência de parto (MEDEIROS et al., 2019).

Frente à transcendência do PP para a autonomia da gestante e da parturiente, é essencial que a equipe de enfermagem conheça esse instrumento, visando aperfeiçoar a assistência dispensada a esse público, iniciando a confecção do PP durante o pré-natal. Os enfermeiros atuantes na atenção primária à saúde precisam estar atentos às novas diretrizes do MS para que o PP seja inserido no cotidiano da atenção pré-natal, pois a elaboração e utilização do mesmo favorecem a prática do profissional, indo ao encontro da autonomia da gestante no momento do trabalho de parto e parto (MOUTA et al., 2017).

O empoderamento se fortalece no processo educativo, que tem como principal propósito o desenvolvimento de conhecimentos, atitudes, habilidades e autoconhecimento para que, dessa forma, as mulheres assumam de maneira efetiva a responsabilidade com as decisões a serem tomadas no que diz respeito a sua saúde (MOUTA et al., 2017). Através de atividades de educação em saúde, as gestantes obtêm informações fundamentais para a construção das suas concepções próprias, libertas de preconceitos e paradigmas estabelecidos pela evolução da sociedade (PEREIRA et al., 2018).

Um exemplo são as rodas de conversa, nas quais se estimula que as gestantes possam compreender e expressar seus valores culturais, medos, necessidades, possibilitando comunicar-se sobre as expectativas e preferências aos profissionais da assistência. Diante disso, o ato de elaborar o PP em rodas de conversa surge como uma estratégia que oportuniza a discussão sobre o parto, trazendo uma 
gama de ações que visam conversa sobre iatrogenias e danos relacionados a métodos dispensáveis realizados habitualmente com a mulher e o bebê na cena do parto (PEREIRA et al., 2018).

Para estarem seguras em relação às decisões concernentes a esse momento especial em suas vidas, as gestantes precisam captar um aprendizado precedente sobre o assunto. Para tanto, os enfermeiros da atenção básica, durante o pré-natal, devem fornecer às mulheres, além das informações sobre a gestação, referências sobre o processo parturitivo por completo. A roda de conversa como estratégia de construção do PP é um dos métodos mais indicados para ser utilizado pelos profissionais (SILVA et al., 2015).

Além disso, destaca-se o uso de tecnologias da informação em saúde. Esse uso é considerado uma estratégia capaz de melhorar a qualidade da assistência ofertada às gestantes, melhorar a continuidade dos registros, incentivar a completude dos dados, fornecer apoio à decisão e reduzir a incidência de erros clínicos na área obstétrica. Uma vez que, os registros em papel podem conter limitações importantes capazes de dificultar a continuidade do cuidado (dados ilegíveis e/ou incompletos, não padronizados e/ou inválidos), isso desfavorece o acesso aos dados para pesquisa ou avaliação de um serviço (CARILHO et al., 2016).

\section{CONCLUSÃO}

A construção do PP durante o pré-natal estimula de maneira positiva o protagonismo da mulher e de sua família no processo parturitivo, bem como corrobora com melhores desfechos materno-fetais. Destacam-se os benefícios desse instrumento, o qual possibilita promover um processo de parto mais natural e fisiológico, a melhor comunicação com profissionais da saúde e a maior conscientização das gestantes em relação aos eventos envolvidos no trabalho de parto e parto. Isso resulta na sensação de maior controle e consequentemente maior grau de satisfação materna.

Dentro do contexto em que foi estudado, o PP pode ser considerado uma ferramenta educativa, pois busca a organização de informações relativas à assistência do parto de duas maneiras: com o reconhecimento de que procedimentos desnecessários podem afetar a segurança e bem-estar da mãe e do bebê e dos direitos e de situações de violência.

O envolvimento e o apoio dos profissionais capacitados e sensibilizados são imperiosos na elaboração e efetivação do PP. Para tanto, eles devem buscar mais conhecimento no que diz respeito ao PP como forma de oferecer às gestantes um maior suporte na orientação da construção do instrumento. Assim como, os profissionais devem disponibilizar estratégias para a elaboração do plano, não contemplando somente a ocasião da consulta de pré-natal, mas possibilitando de construção individual e/ou coletiva.

Observa-se que os enfermeiros necessitam conhecer a realidade da rede de atenção à saúde materno infantil dentro de cada município a fim de orientar práticas condizentes com as possibilidades de infraestrutura e recursos humanos das instituições de saúde. Assim como, fomentar a comunicação entre a atenção primária à saúde e às maternidades de referência a fim de trabalharem na mesma linha 
de cuidado ofertado. Ainda, ressalta-se a importância da atualização dos profissionais de saúde e a incorporação de novas práticas dentro das rotinas das instituições de saúde nos diferentes eixos de atenção.

Com a realização deste estudo, foi notória a necessidade da realização de mais pesquisas e publicações acerca do tema, como forma de instruir e ofertar embasamento científico para os enfermeiros no que tange às estratégias de construção do PP. Além disso, reforçar-se a necessidade da inserção desse instrumento nos protocolos de atenção básica como parte da rotina das consultas de pré-natal, a fim de proporcionar uma melhor qualidade na assistência para a gestante e sua família.

\section{REFERÊNCIAS}

BARROS, Amanda Peres Zubiaurre de et al. Conhecimento de enfermeiras sobre o plano de parto. Revista de Enfermagem da UFSM. v. 7, n. 1, p. 69-79, 2017.

BRASIL. Ministério da Saúde. Diretrizes nacionais de assistência ao parto normal: versão resumida. Secretaria de Ciência, Tecnologia e Insumos Estratégicos. Departamento de Gestão e Incorporação de Tecnologias em Saúde - Brasília: 2017. Disponível em: https://bit.ly/3kcF3il. Acesso em: jan. 2020.

BRASIL. Ministério da Saúde. Caderno HumanizaSUS: humanização do parto e do nascimento. Universidade Estadual do Ceará - Brasília: 2014. Disponível em: https://bit.ly/32uWXHc

BRASIL. Ministério da Saúde. Portaria $n^{0}$ 1.459, de 24 de junho de 2011. Institui no âmbito do Sistema Único de Saúde - SUS - a Rede Cegonha. 2011. Disponível em: https://bit.ly/36jA3DT

BRASIL. Ministério da Saúde. Programa Humanização do Parto: Humanização no pré-natal e nascimento. Secretaria Executiva - Brasília: 2002. Disponível em: https://bit.ly/2I1NNpC

BRUM, Crhis Netto et al. Revisão narrativa da literatura: aspectos conceituais e metodológicos na construção do conhecimento da enfermagem. In: LACERDA, Maria Ribeiro; COSTENARO Regina Gema Santini. Metodologias da pesquisa para enfermagem e saúde da teoria à prática. v. 1, n. 1, 2016.

CARRILHO Juliane Moraes et al. Proposição do plano de parto informatizado para apoio a interoperabilidade e humanização. XV Congresso Brasileiro de Informática em Saúde - CBIS 2016. Disponível em: https://bit.ly/2IoiPN4.

CORTÉS, Maria Suarez et al. Uso e influência dos planos de parto e nascimento no processo de parto humanizado. Revista Latino-Americana de Enfermagem. v. 23, n. 3, p. 520-6, 2015. 
GOMES Rebeca Pinto Costa et al. Plano de parto em rodas de conversa: escolhas das mulheres. Revista Mineira de Enfermagem. v. 21: e-1033, 2017.

GONÇALVES, Maria Faria et al. Pré-natal: preparo para o parto na atenção primária à saúde no sul do Brasil. Revista Gaúcha de Enfermagem. v. 38, n. 3, p. 2016-0063, 2017.

GUEDES, Cintia Danielle Faustino da Silva et al. Percepções de gestantes sobre a promoção do parto normal no pré-natal. Revista Ciência Plural. v. 3, n. 2, p. 87-98, 2017.

LEAL, Maria do Carmo et al. Intervenções obstétricas durante o trabalho de parto e parto em mulheres brasileiras de risco habitual. Cadernos de Saúde Pública. v. 30, suppl. 1, p. 17-32, 2014.

MEDEIROS, Renata Marien Knupp et al. Repercussões da utilização do plano de parto no processo de parturição. Revista Gaúcha de Enfermagem. v. 40, n. 20, p. 180233, 2019.

MOUTA, Ricardo José Oliveira et al. Plano de parto como estratégia de empoderamento feminino. Revista Baiana de Enfermagem. v. 31, n. 4, p. 1-9, 2017.

NARCHI, Nádia Zanon et al. O plano individual de parto como estratégia de ensino-aprendizagem das boas práticas de atenção obstétrica. Revista Escola de Enfermagem da USP. v. 53, n. 3, p. 1-9, 2019.

PEREIRA, Simone Barbosa et al. Boas práticas de atenção ao parto e ao nascimento na perspectiva de profissionais de saúde. Revista Brasileira de Enfermagem. v. 71, n. 3, p. 1313-9, 2018.

SANTOS, Fernanda Soares de Resende et al. Os significados e sentidos de plano de parto para as mulheres que participaram da exposição Sentidos do Nascer. Cadernos de Saúde Pública. v. 35, n. 6, e-00143718, 2019.

SILVA, Adaiele Lucia Nogueira Vieira et al. Plano de parto: ferramenta para o empoderamento de mulheres durante a assistência de enfermagem. Revista de Enfermagem da UFSM. v. 7, n. 1, p. 144-151, 2017.

SILVA, Stefane Gomes et al. Perfil de gestantes participantes de rodas de conversa sobre plano de parto. Enfermagem Obstétrica. v. 2, n.1, p. 9-14, 2015.

World Health Organization. WHO recommendations: intrapartum care for a positive childbirth experience, 2018. Disponível em: https://bit.ly/2UbZ8ed 
